\title{
Médiévales
}

Langues, Textes, Histoire

67 | automne 2014

Histoires de Bohême

\section{Représentations de l'intimité dans le roman arthurien tchèque}

Centrum medievistických studií AV ČR (Centre d'études médiévales), Prague.

Representations of Intimacy in the Czech Arthurian Romance

\section{Martin Šorm}

\section{(2) OpenEdition}

\section{Journals}

Édition électronique

URL : https://journals.openedition.org/medievales/7385

DOI : 10.4000/medievales.7385

ISSN : 1777-5892

Éditeur

Presses universitaires de Vincennes

\section{Édition imprimée}

Date de publication : 31 décembre 2014

Pagination : 49-66

ISBN : 978-2-84292-422-5

ISSN : 0751-2708

Référence électronique

Martin Šorm, «Représentations de l'intimité dans le roman arthurien tchèque », Médiévales [En ligne],

67 | automne 2014, mis en ligne le 31 décembre 2016, consulté le 22 avril 2022. URL : http://

journals.openedition.org/medievales/7385; DOI : https://doi.org/10.4000/medievales.7385 
Martin Šorm

\section{Représentations de l'intimité dans le roman arthurien tchèque}

La littérature médiévale tchèque est souvent présentée comme peu ou, même, «pas assez» courtoise. On utilise en général pour la définir des attributs comme «réaliste», «terre-à-terre» ou «simpliste», avec un fort accent «moraliste», proche du «quotidien», éloignée des idéaux de l'aristocratie et plutôt destinée aux couches moyennes, voire bourgeoises ${ }^{1}$. Bien que certains de ces jugements soient fondés sur des analyses érudites des textes de la fiction médiévale tchèque, la plupart d'entre eux découlent surtout de l'effort d'éclairer, dans sa singularité, le développement social et politique inattendu du royaume de Bohême au $\mathrm{Xv}^{\mathrm{e}}$ siècle. D'après la composition des manuscrits contenant les deux romans arthuriens tchèques, Tristram a Izalda et Tandariáš a Floribella, il semble pourtant évident qu'ils ont été plutôt lus dans les milieux aristocratiques, ce qui remet en question les jugements que nous avons précédemment mentionnés.

Il semble difficile d'incorporer la fiction littéraire dans la conception globale de l'histoire tchèque du Moyen Âge tardif. Cela résonne avec les éternels débats sur les origines de la chevalerie et de la culture courtoise de la noblesse en Bohême ${ }^{2}$. On s'accorde maintenant sur le fait que, pendant la deuxième moitié du XII ${ }^{\mathrm{e}}$ siècle, avec les campagnes militaires de Vladislav II et Conrad II Ota en Terre sainte et en Italie, une aristocratie

1. Cf. A. Thomas, Annes's Bohemia: Czech Literature and Society, 1310-1420, Minneapolis, 1998, p. 110-124; mais aussi H. VoISINE-JECHOVA, «Tristram et Izalda. Notice», dans Tristan et Yseut: les premières versions européennes, éd. C. MARChello-NiziA, Paris, 1995, p. 1600-1603. Pour la critique de cette approche, voir J.HoN, «Jetřich Berúnský: "Dramatizace" středohornoněmeckého Laurina", dans B. Hanzoví éd., Pokušení Jaroslava Kolára, Prague, 2009, p. 31-52.

2. J. МАCEK, Česká středověká šlechta, Prague, 1997; W. IwAŃCZAK, Tropem rycerskiej przygody: Wzorzec rycerski w pismiennictwie czeskim XIV wieku, Varsovie, 1985 ; R. ANTONíN, Ideální panovník českého středověku, Prague, 2013. 
chrétienne avec des domaines féodaux et des liens internationaux s'est formée et s'est développée, au point de pouvoir briller dans les tournois du XIII ${ }^{\mathrm{e}}$ siècle, construire ses églises et ses châteaux forts, et même soutenir des poètes allemands, dont l'activité est documentée non seulement à la cour des derniers Přemyslides, mais aussi auprès de plusieurs familles nobles.

On observe néanmoins une forte tendance à mettre en doute l'impact de la culture chevaleresque sur la noblesse tchèque au temps de Jean de Luxembourg (1310-1346), bien que celle-ci semble vivre au XIV ${ }^{\mathrm{e}}$ siècle son plein essor. Tout se passe comme si la courtoisie, avant la révolution hussite, devait être introduite en Bohême d'une façon inorganique ${ }^{3}-$ ou plutôt comme si les médiévistes eux-mêmes ne voulaient pas l'intégrer dans leur vision du «Moyen Âge tchèque». Cela dit, les cinq manuscrits contenant les récits que l'on va étudier ici datent de la deuxième moitié du $\mathrm{XV}^{\mathrm{e}}$ siècle ( $\left.c a 1450-1480\right)$, donc après les guerres hussites. Les œuvres ellesmêmes ont été composées probablement sous le règne de Venceslas IV (1378-1419), ce qui nous permet de supposer qu'elles étaient populaires durant toute cette période intermédiaire ${ }^{4}$.

Parallèlement, on a longtemps considéré les œuvres littéraires en vieux tchèque comme de simples traductions de «romans-modèles » allemands, qui ne méritaient donc que peu d'intérêt. L'approche prédominante a privilégié les comparaisons - soit impitoyables, soit apologétiques - avec des versions allemandes plus anciennes. Le but était d'expliquer - ou plutôt de justifier - le raccourcissement radical du texte originel par le fait que le poète anonyme tchèque avait cru nécessaire d'adapter la matière du récit à un auditoire prétendument non accoutumé à de longs monologues amoureux ou psychologisants. Les explications des changements d'expression et de style se fondent souvent sur des a priori concernant les caractéristiques des auditeurs tchèques. Enfin, on essayait de dissimuler les prétendues faiblesses du texte tchèque: on accentuait alors l'ingéniosité avec laquelle le traducteur-auteur tchèque avait introduit quelques nouveaux motifs dans son récit ${ }^{5}$.

3. Cette expression est utilisée par Y.Millet dans sa recension d'U.BamborschKe, Der alť̌echische Tandariuš nach den 3 überlieferten Handschriften mit Einleitung und Wortregister, Wiesbaden, 1982, Revue des études slaves, 55 (1983), p. 389-390.

4. E. Petrư, Vzdálené hlasy.Studie o starší české literatuře, Prague, 1996, p. 71-80; ID., «Rytî́ský epos a jeho proměny», dans D. MarečKová et E.PeTrư éd., Rytiřrské srdce majice. «Rytî́ský epos a jeho promèny», dans D.MAREČKOVÁ
Česká rytî́rká epika 14. století, Prague, 1984, p. 7-22.

5. On rappelle ainsi souvent la virtuosité de l'auteur de Tristram a Izalda. Ce dernier a développé l'idée d'Eilhart d'Oberg selon laquelle Tristran, fugitif dans la forêt, a résolu le problème de l'approvisionnement en nourriture en inventant la pêche à la ligne avec l'hameçon. Le poète tchèque accentue surtout l'assistance essentielle de son amie. Voir A.Thомas, «Czech Arthurian Literature», dans N. J. LaCY éd., The New Arthurian Encyclopedia, New York-Londres, 1991, p. 106-108. Ce passage, accompagné par deux autres, représente la version tchèque de Tristan dans l'édition de La Pléiade: H. Vorsne-
Bien que la fiction médiévale tchèque soit aujourd'hui considérée comme une source historique, elle reste toujours négligée par les historiens du Moyen Âge. Il faut mentionner encore un autre élément important: la littérature courtoise tchèque (surtout Jetřich Berúnský ${ }^{6}$, en plus des œuvres que l'on appelle ici «arthuriennes») n'est presque jamais concernée par la conversion ou l'ascension vers une chevalerie spirituelle. Toujours écrite en vers et principalement considérée comme amusante, elle n'a presque rien en commun avec les cycles allégoriques français en prose du XIII ${ }^{\mathrm{e}}$ siècle. En outre, il n'y a aucune mention du Graal dans les sources de la Bohême médiévale. Ce phénomène, difficile à expliquer, décontenance également les chercheurs qui mettent en valeur le caractère profondément religieux de la société tchèque de l'époque et son ouverture à une réforme spirituelle sans précédent.

Les sources littéraires tchèques des $\mathrm{XIV}^{\mathrm{e}}$ et $\mathrm{XV}^{\mathrm{e}}$ siècles nous lancent un défi exceptionnel : elles enrichissent fondamentalement notre perception de la culture de la noblesse tchèque pendant les guerres hussites. On commence à comprendre que, pour les nobles, acquis à la réforme ou non, le mouvement réformateur et les différences confessionnelles n'empêchaien pas de perpétuer dans une pleine continuité les festivités traditionnelles ni d'entretenir de bonnes relations au sein des lignées nobles, pourtant divisées pour des raisons confessionnelles ${ }^{7}$. La rupture n'a pas touché non plus le goût pour l'imaginaire chevaleresque arthurien. Les récits ne traitent pas des questions religieuses. Leur objectif semble être de cultiver et de raffiner la sensibilité du public, de manière divertissante, discrète (pour ne pas susciter le rejet des auditeurs), mais toujours élaborée. Dans ce sens, ils n'enseignent pas la courtoisie différemment des autres romanciers médiévaux.

Je vais me concentrer ici sur les aspects des romans arthurien tchèques qui remettent en cause les jugements méprisants parfois émis à leur égard et qui tendent à les exclure de la littérature courtoise ${ }^{8}$. La culture courtoise était toujours recréée et reconstituée par des auteurs et leurs textes. Ces derniers ont élaboré des représentations de la nature absolue d'un amour considéré comme source de perfectionnement. Ils se sont efforcés de façonner leur public par un choix de sujets subtils, par les manifestations de doutes et d'hésitations de chevaliers fictifs. Ils posent avant tout des questions et ne répondent pas toujours à celles-ci. Leurs récits, outre leur

Jechova éd., Tristram et Izalda. Quatre épisodes du roman tchèque, dans Tristan et Yseut... éd. C. MarCHELlo-NIzia, p. 1107-1123 (ici p. 1112).

6. Aussi connu comme Malá růžová zahrada («Le petit jardin des roses»), dan D. MareČKová et E.Petrư éd., Rytî́rské srdce majíce...., p. 195-256.

7. R. NovotnÝ et P. SoukuP, «La défense de la foi à l'époque hussite: l'engagement de la noblesse tchèque et allemande», dans A.BoltansKi et F. MERCIER éd., Noblesse et défense de l'orthodoxie (XIII'-XVII' siècles), Rennes, 2011, p. 93-108.

8. Comme le fait A. THомаs, Annes's Bohemia ...p. 110. 
visée distrayante, poursuivaient également un objectif d'instruction morale et de raffinement du comportement. En dévoilant des tensions internes de la société aristocratique, les romanciers ont suscité et cultivé des réflexions importantes. Ces tensions se révèlent de manière exemplaire dans ce que j'appellerais la représentation de l'intériorité et de l'intimité.

Par «l'intime», je ne comprends pas seulement les images des relations interpersonnelles les plus profondes et les plus importantes, mais aussi la construction mentale des personnages - souvent représentés comme incertains, faibles, renfermés sur eux-mêmes voire au bord de la folie. Plutôt qu'à des représentations de la consommation de l'amour, on va se référer surtout, ici, aux images littéraires de l'oubli de soi, de la réflexion profonde et de l'autodestruction chevaleresque - tout en tenant compte des aspects performatifs de l'intime. Je voudrais insister plus précisément sur le motif de l'intimité du roi comme préoccupation partagée par toute la société, ce qui suscite une tension entre privé et public manifestée en particulier par le symbole du lit royal, présent dans l'ensemble des récits arthuriens médiévaux.

\section{Le piège courtois de Floribella, la main entaillée et l'oubli de Tandariáš}

Le premier des deux romans que l'on va étudier ici est conservé dans trois manuscrits de la deuxième moitié $\mathrm{du}^{\mathrm{x} v^{\mathrm{e}}}$ siècle. Il s'y trouve en compagnie d'autres œuvres de fiction, mais aussi de textes représentant les intérêts politiques de l'aristocratie tchèque de la fin du Moyen Âge ${ }^{9}$. L'hypotexte,Tandareis und Flordibel de Pleier, poète autrichien du XIII ${ }^{\mathrm{e}}$ siècle, est presque dix fois plus long que la version tchèque ${ }^{10}$. Les quelque deux mille vers en vieux tchèque racontent l'histoire d'une demoiselle indienne à la cour du roi Arthur (Artuš) et de son chevalier protecteur qui devient son amant et, à la fin du récit, son heureux mari. À part qu'elle est «indienne», on ne sait rien sur les origines de Floribella, sinon qu'elle est orpheline. Lors de son arrivée, elle dicte les «règles du jeu» de son séjour à la cour. Elle exige un serment d'Arthur qui lui assurerait sa propre chasteté. Le roi devra décapiter chaque homme qui osera mettre en cause l'honneur de la princesse. L'auteur mentionne explicitement la possibilité qu'elle soit ellemême sujette à des tentations charnelles. Par ce moyen, il crée l'attente

9. Surtout le Nouveau conseil de Smil Flaška de Pardubice ou la chronique de Dalimil. Voir M.NEJEDLÝ, «L'idéal du roi en Bohême à la fin du quatorzième siècle. Remarques sur le Nouveau conseil de Smil Flaška de Pardubice», dans D.Boutet et J. Verger éd., Penser le pouvoir au Moyen Âge : études offertes à Françoise Autrand, Paris, 2000, p. 247-260.

10. Pour une comparaison solide et approfondie, voir V. E. MoureK, Tandariuš a Floribella. Skládání staročeské sněmeckým Pleierovým, Prague, 1887. d'une potentielle transgression. Arthur choisit Tandariáš qui devra la servi «sans aucun abus ${ }^{11} »$.

Le roman décrit alors comment Floribella et son chevalier tombent tous deux dans ce piège que la princesse elle-même avait tendu en obligeant toute la cour à veiller sur sa propre intimité. Amoureux, ils fuient Arthur qui avait pourtant juré de la protéger - y compris en défendant son enlèvement. Le roi en colère est alors forcé par son serment de les poursuivre, d'assiéger le château du père de Tandariáš où les amants se sont réfugiés, et de voir ses meilleurs chevaliers, Gauvain (Gawin) et Keu (Kajn), vaincus. Incapable d'accomplir ses promesses, il décide de faire Floribella prisonnière, bien qu'elle ait maintenant essayé en vain de mettre fin au jeu qu'elle avait pourtant si ingénument arrangé ${ }^{12}$. Tandariáš est expulsé en revanche de la cour, ce qui est une occasion pour le romancier de le soumettre à une série d'aventures diverses et inouïes ${ }^{13}$. Malheureusement, au moment où Arthur lui pardonne enfin et le rappelle à la cour, Tandariáš est lui-même emprisonné par une autre princesse, désespérément amoureuse de lui, et par son méchant frère, ce qui tient en haleine Floribella, la cour arthurienne, mais aussi ses lecteurs ou ses auditeurs. La fin joyeuse du roman dépein cependant le mariage des deux protagonistes dans une tonalité tellement gaillarde qu'elle nous rappelle un peu l'explication des origines des eunuques byzantins à la fin du roman de Cligès: Arthur a non seulement marié Tandariáš et Floribella, mais il a aussi trouvé une naine convenable pour un nain qui avait autrefois menacé Tandariáš avec ses deux léopards en laisse.

Toute l'histoire est racontée dans une langue légère et ironique. L'intrigue courtoise est présentée comme un jeu divertissant. Toutefois, le romancier montre beaucoup d'intérêt pour les mouvements et les haltes profondes de l'esprit de Tandariáš. Celui-ci se montre non seulement excellent chevalier, mais aussi oublieux ${ }^{14}$, pensif $^{15}$, passif - ce qui signifie que, outre une maîtrise active du monde, il sait également éprouver, douter,

11. Tandariáš a Floribella, dans D. MAREČKovÁ et E.Petrư éd., Rytiřské srdce majice.... p. 256-309 (ici v. 80-87 et v.112).

12. Elle reçoit l'appui de Gauvain qui, trait typique de ce personnage, essaie de persuader Arthur d'annuler son serment pour favoriser le retour du calme social (ce passage est comparable au début d'Érec et Énide, v. 39-62: éd. J.-M. Fritz, dans ChrétIEN DE Troyes, comparable au début d'Erec et

13. Soulignons qu'il n'y a aucun équivalent fixe en vieux tchèque pour l'expression «aventure»: voir J.Hon, «Jetřich Berúnský... », p. 35.

14. M. SzKILNIK, «Le chevalier 'oublieux' dans le roman arthurien en vers», dans P. Romagnoli et B. Wahlen éd., Figures de l'oubli (IXe'-XVI' siècle), Lausanne, 2007, p. 77-97; J. RibARD, «Amour et oubli dans les romans de Chrétien de Troyes», dans D.Quéruel éd., Amour et chevalerie dans les romans de Chrétien de Troyes, Paris, 1995, p. 83-94.

15. Ce qu'apprécie Gauvain dans Le Conte du Graal comme un signe de «courtoisie et douceur» (éd. C. Méla, dans ChrétIEn de Troyes, Romans, p. 1072, v. 4389-4393). 
être défaillant, se faire guider, se faire blesser, croire, et devenir ainsi pareil à l'un des «chevaliers humiliés» dont Michel Zink a particulièrement souligné l'importance ${ }^{16}$.

Attachons-nous maintenant à étudier l'épisode de la «main entaillée ». Au début du récit, devenu protecteur et serviteur de la belle Indienne, Tandariáš se hâte chaque matin d'arriver plus tôt que les chambellans devant la porte de sa chambre avec un récipient doré rempli d'eau. Floribella, flattée, modifie ses habitudes: elle se lève encore plus tôt afin qu'ils puissent jouir de ces moments d'intimité. Ainsi, le chevalier n'exprime son affection que par sa présence presque incessante et toujours silencieuse, puisqu'il est conscient de la menace de l'exécution. Une fois cependant, à genoux et tranchant le pain, il réfléchit et la regarde, assise à la table ${ }^{17}$ :

[...] il leva les yeux et donc il commença à s'oublier lui-même et à s'entailler la main. Floribella poussa un cri et dit: «Attention à ta main!» Il regarda sa main et sortit en courant pour la laver et la panser. Puis il revint auprès de la demoiselle et recommença à la servir. Elle se demanda pourquoi il n'éprouvait aucune sensation de soi [...]. Elle l'interrogea d'une voix douce: «Lève-toi et raconte-moi ce que tu as pensé. Pour quelle raison n'as-tu eu aucune sensation de toi quand tu t'es coupé la main? À quoi pensais-tu à ce moment-là, lorsque tu étais ici devant la table, en t'oubliant toi-même?»

Les questions insistantes de Floribella mènent à l'expression mutuelle d'un amour aussi passionné que borné par un sens de l'honneur qui empêche les protagonistes de le manifester ouvertement. La manière de formuler l'état amoureux et la timidité du meilleur chevalier de la cour nous rappelle Lancelot et ses membres entaillés par le pont de l'épée ou par les barreaux de la fenêtre de la reine. Rappellons également l'oubli de soi qui frappe les chevaliers amoureux, soit près d'un gué, soit en contemplant des gouttes de sang sur la neige ${ }^{18}$. J'insiste sur le fait que toutes mes comparaisons occasionnelles de certains passages du roman tchèque avec ceux des œuvres de Chrétien de Troyes (et on pourrait étendre ces comparaisons à bien d'autres romans français, notamment avec Jehan et Blonde de Philippe de Rémi,

16. Voir ses lectures au Collège de France des années 2010-2012: «Humbles et humiliés. Récits médiévaux de l'abaissement» (http://www.college-de-france.fr/site/michel-zink/ course-2011-12-07-10h30.htm).

17. Tandariáš a Floribella, p. 263, v. 140-163. Les traductions du vieux tchèque sont dues à l'auteur de cet article.

18. Chrétien de Troyes, Le Chevalier de la Charrette,v. 3100-3141, 4636-4646, 736-771 (éd. C. Méla, dans Romans, p. 589-590, 633, 521-522); ID., Le Conte du Graal, v. 4133-4149 (Romans, p. 1065). contemporain de Pleier ${ }^{19}$ ), n'ont ici certainement pas pour objectif d'indiquer une relation filiale directe entre ces textes particuliers. Je cherche plutôt à faciliter notre compréhension des situations littéraires moins connues en les mettant en parallèle avec celles qui ont été déjà plusieurs fois et minutieusement étudiées grâce à la célébrité actuelle du poète champenois . L'histoire de Tandariáš doit être ainsi replacée dans le contexte européen de la littérature médiévale avec sa polysémie textuelle. En ce sens, je peux me permettre sans aucun doute d'assimiler également la maladie prétendue de Floribella, par laquelle elle essaye d'esquiver sa présence annuelle à la cour et d'échapper avec Tandariáš à Arthur, à la fausse mort de Fénice dans Cligès $^{20}$.

De ce point de vue, il faut préciser qu'apparemment, à la différence du Chevalier à la Charrette ou au Conte du Graal, le récit des épreuves de Tandariáš ne semble pas avoir une dimension spirituelle considérable. Le sang, qui n'est même pas explicitement mentionné par le romancie tchèque, ne coule de sa main qu'à cause de sa fascination pour la femme aimée. Cependant, la fonction de Tandariáš dans le texte est comparable à celle d'un Lancelot du Lac dépourvu de sa dimension salvatrice et presque christique.

Chaque oubli de soi met en cause la conception du chevalier comme masculinité incarnée: un héros toujours conscient et actif dans sa lutte perpétuelle contre le mal $^{21}$. Il prouve également son aptitude à se donner, sa capacité de passion. Si un tel état d'inconscience, qu'il s'agisse d'un oubli de soi à la limite de l'extase mystique ou d'une simple réflexion profonde (ce qui est plutôt le cas de Tandariáš), est devenu un motif important de la littérature arthurienne médiévale, il faut s'interroger sur sa signification. Ne signale-t-il pas, entre autres, une certaine tension dans la société médiévale par rapport à l'exigence générale de responsabilité et de performance comme ciment des hiérarchies profanes et comme fondement de toute communication sociale? Rappelons ici le grand scandale décrit soigneusement par Chrétien de Troyes, provoqué par Arthur au début même du roman du Chevalier au Lion, quand, après avoir quitté ses chevaliers, le roi «s'oblia et s'endormi »dans la chambre de la reine au lieu de deviser avec eux dans la salle ${ }^{22}$ ? Cette scène ne montre-t-elle pas explicitement un conflit entre l'intimité du roi et son devoir public? Je reviendrai sur cette

19. PHILIPPE DE RÉmi, Jehan et Blonde, roman du XIII siècle, éd. S. LÉCUYER, Paris, 1984 p. $39-40$, v. $425-454$

20. Chrétien de Troyes, Cligès, v. 5264-5277 (Romans, p. 450).

21. Voir les réflexions sur daydream et social usefulness du chevalier chez R.Pensom, Aucassin et Nicolette. The Poetry of Gender and Growing Up in the French Middle Ages, Berne, 1999, p. 37-39.

22. Chrétien de Troyes, Le Chevalier au Lion, v. $42-52$ (éd. D. F. Hult, dans Romans, p. 712-713). 
critique en interprétant quelques scènes du roman de Tristram a Izalda. Pour l'heure, je soulignerai surtout «l'oubli» du roi dans sa chambre. Arthur a vécu un oubli de soi, de sa condition, de ses obligations, de son pouvoir. Le romancier sent le besoin d'exprimer une telle possibilité, ce qui témoigne de sa recherche intense d'un équilibre entre le bonheur privé du roi et de son devoir envers la société.

C'est également un oubli de soi et une réflexion profonde qui mettent en danger tout l'entourage de Tandariáš lorsque ce dernier se plonge dans le souvenir de son amie ${ }^{23}$ :

Ils chevauchèrent ainsi assez longtemps. Une fois dans une immense forêt, Tandariáš s'emplit d'un désir passionné. Il pria donc les autres d'avancer seuls en disant: «Je veux rester un peu en arrière et me souvenir de ma bien-aimée. Il me faut faire passer le temps car je souffre beaucoup.» Il les suivit donc sans se presser jusqu'à ce qu'il s'éloigne d'une demi-lieue, en se rappelant les temps passés où lui et son amie se réjouissaient d'être ensemble. À ce moment-là, ceux qui étaient en avance furent assaillis par une troupe de brigands. Avant que Tandariáš ne puisse arriver pour les aider, les assaillants avaient commencé à les capturer.

Voilà les conséquences de ce repli intérieur - intéressant pour son intentionnalité, voire pour sa planification consciente. D'une part, l'auteur s'efforce ici d'éduquer son public et de raffiner sa sensibilité en soulignant l'importance du souvenir et du libre flux des pensées. Mais, d'autre part, il souligne les difficultés liées à tout éloignement du monde - soit physique, soit mental. Dans l'ensemble du roman, particulièrement court par rapport aux autres récits arthuriens médiévaux, le poète concentre un grand nombre de situations où le chevalier est décrit comme inactif, soit parce qu'il intériorise consciemment des images visuelles, soit parce qu'il est inattentif et submergé par ses sentiments. C'est aussi le cas d'une des scènes finales où, encore une fois, pas très différemment de Lancelot, Tandariáš quitte à plusieurs reprises sa prison pour pouvoir participer à un tournoi ${ }^{24}$. Le visage couvert, il réussit à désarçonner son ravisseur, qui participe lui aussi à ce tournoi. La reine croit le reconnaître et se dépêche de l'annoncer à Floribella. Celle-ci reste cependant dans sa chambre et refuse de prendre part aux festivités collectives - triste et «langoureuse, passant son temps à lire des livres ${ }^{25} \gg$. La cour se réunit à nouveau quelques jours plus tard et tout se répète: Tandariáš, chevalier inconnu, revient et bat de nouveau le prince dont il est prisonnier. Cette fois, Floribella se reproche de ne pas

23. Tandariáš a Floribella, p. 272 , v. 460-478.

24. Ibid., p. 294-304, v. 1285-1673.

25. Ibid., p. 297 , v. $1401-1403$ et p. 298 , v. 1449. avoir assisté à la scène, malgré l'insistance de la reine ${ }^{26}$. Enfin, lors de la troisième assemblée, elles vont toutes les deux regarder le tournoi où Tandariáš sera également présent, désireux d'apercevoir son amie ${ }^{27}$. Ses yeux le lui permettent ${ }^{28}$.

Il fut si joyeux qu'il ne se rendit pas compte de ce qu'il faisait. Peu importe qui il croisait, il le frappait d'estoc ou de taille. [...] Il ne cesse pas de regarder son amour de telle manière qu'il s'oublie lui-même. Pour cette raison, il reçoit plusieurs coups. Il reste planté comme un piquet, le regard sans cesse fixé sur la fenêtre. Un de ses compagnons lui crie alors: «Reviens à toi!» Ce n'est qu'après cela qu'il prit conscience de lui-même et recommença à donner lui aussi des coups

Après le départ de son ami, Floribella a tant de chagrin que le sang coule de son nez et de sa bouche. Elle ne revient pas à elle-même avant que la reine ne parvienne à la rassurer. C'est la seule fois dans ce roman qu'une femme subit une perte de conscience - rappelons qu'on ne parle pas ici d'évanouissement, dont on trouve beaucoup de cas explicites dans la fiction médiévale et qui ne concernent pas principalement les femmes. La manifestation physique, sanglante, qui accompagne cet oubli de soi féminin, représente l'humidité essentielle du corps féminin ${ }^{29}$. Elle constitue en outre un pendant narratif aux blessures dont souffre Tandariáš - soit dans la bataille, soit en tranchant le pain, enivré qu'il est par son profond amour.

En ce qui concerne l'importance de l'amie du chevalier dans ses triomphes, il me semble nécessaire de mentionner encore une autre scène caractéristique de l'histoire de Tandariáš et Floribella. La perception subjective de la voix de son amie (même si elle est illusoire) permet au chevalier de regagner ses forces et de reprendre le dessus. Pendant une lutte violente contre un païen ${ }^{30}$, Tandariáš entend des voix féminines lui

26. Ibid., p. 300-301, v. 1505-1539.

27. Ibid., p. 302-304, v. 1589-1657.

28. Ibid., p. 303, v. 1614-1629.

29. Ibid., p. 304, v. 1636-1644. À propos du saignement de nez significatif, voir par exemple une pareille situation dans le roman Cleriadus et Meliadice. Roman en prose du XV' siècle, éd. G. ZiNK, Paris-Genève, 1984, p. 376. Il s'agit d'un cas typique de construction sociale du corps féminin : le saignement est ici une expression de la sensibilité incontrôlable et excessive. Il accompagnait par exemple des extases mystiques, mais servait aussicon exces comme représentation littéaire de la menstrution - le corps féninin a souvent été perçu comme plus froid et humide. Voir J.-M.FRITZ, «La theorie humorale comme moyen de penser le monde. Limites et contradictions du système », dans D. Boutet et L. HARF-LANCNER, Écriture et modes de pensée au Moyen Âge (XIII'-XV' siècles), Paris, 1993, p. 13-26.

30. Une certaine indifférence religieuse dans le roman courtois tchèque se manifeste bien à propos de ce païen - une fois battu, on ne parle plus du tout de la possibilité de sa conversion. L'auteur ne s'en préoccupe pas, contrairement à ce que peut observer C.Girbea dans les romans allemands: C. GirBEA, «Le double romanesque et la conversion. Le Sarrasin, 
rappelant celle de Floribella. Le fait qu'il se soit alors «souvenu de sa bienaimée ${ }^{31} \gg$ l'a sauvé d'une défaite mortelle imminente et il lui a aussi assuré la victoire finale.

Il y a donc dans ce récit beaucoup d'images littéraires qui représentent la conscience aussi bien que l'inconscience individuelles: des moments qui renvoient aux situations courtoises traditionnelles, dont celles qui montrent l'importance de l'intériorité profonde - en rapport avec soi et avec son amour. Je ne voudrais toutefois pas donner l'impression que ce combattant fictif est un chevalier hypersensible, toujours souffrant et absorbé dans ses pensées. Voyons par exemple comment il est traité dans la prison où il doit revenir après chaque tournoi. Ces épisodes, eux aussi, concernent directement la représentation de l'intimité. Éprise de Tandariáš, la sœur du méchant prince l'autorise à participer au tournoi, bien qu'il y blesse chaque fois grièvement son frère (qui ne révèle pourtant jamais l'identité de son captif). Cette prison, tout en rappelant les prisons romanesques où des fées gardent des amants, n'est cependant pas conçue par le romancier comme une épreuve de fidélité pour Tandariáš. Celui-ci ne semble pas du tout tourmenté par la présence trop proche d'une autre femme, qu'il n'aime pas, mais qu'il n'essaie pas non plus d'éviter. Il consent même à devenir son chevalier et lui promet fidélité - bien que ce soit seulement un prétexte pour pouvoir sortir de temps en temps et voir sa véritable amie, Floribella. Ce motif n'est pas présenté comme un moment d'hésitation difficile ou d'incertitude. C'est un constat. Le poète ne s'est pas préoccupé de ce qu'on pourrait appeler un manque de résistance de la part du prisonnier courtois. Le récit ne donne aucune réponse aux questions que se pose le lecteur contemporain sur la fidélité à Floribella et les relations que Tandariáš noue avec sa geôlière ne sont pas claires. Après que Tandariáš et le frère de son adoratrice sont revenus l'un après l'autre du tournoi, cette dernière accueille «son chevalier» (comme elle le nomme) de manière - littéralement - très chaleureuse. Elle lui prépare en effet un bain ${ }^{32}$ :

La princesse se réjouissait de le revoir et s'efforçait de le satisfaire. Ensuite, après qu'on lui eut ramené son frère évanoui, à demi-mort, elle ordonna de préparer une cuve où elle ajouta beaucoup d'herbes pour que Tandariáš puisse se baigner. Elle le poussa à s'y asseoir. Pendant qu'il s'y baignait, elle le flagella avec une tige en le réprimandant et en lui reprochant ce qu'il avait fait à son frère: «Pourquoi es-tu si cruel ? Pourquoi as-tu battu mon frère au point qu'il soit maintenant entre la vie et la mort?» Tandariáš riait quand la

32. Ibid., p. 299, v. 1462-1479. princesse lui faisait ces remontrances. Cette scène se répéta plusieurs fois. Voici de quelle façon elle s'est divertie avec son prisonnier.

Quelle que soit la façon dont ils se sont divertis, je doute que le romancie anonyme tchèque soit un grand moraliste. Cela dit, il faut prendre au sérieux ses appels continuels à l'approfondissement et au raffinement de son public. Tout le roman insiste sur la très grande variété des représentations de l'intimité, depuis les conversations et les jeux folâtres jusqu'au dépassement de soi dans la passion amoureuse et à de nombreuses méditations courtoises. Nous avons déjà mentionné un autre élément important dans la représentation de l'intime dans la fiction médiévale, qui figure cependant moins dans le récit de Tandariáš a Floribella que dans celui de Tristram a Izalda: il s'agit d'une tension, souvent conflictuelle, entre le bonheur privé (qui n'est pas seulement la recreantise, selon les termes de Chrétien de Troyes) et l'action publique attendue ${ }^{33}$.

\section{Autour du lit royal: Tristram-sanglier, l'intime et le pouvoir}

Dans la fiction arthurienne, le lit n'est pas seulement une métaphore de l'intime. C'est aussi une représentation du pouvoir, une manifestation de l'autorité et une démonstration de la fortune de celui qui le possède. Tous ces éléments se retrouvent dans une des versions négligées du récit autrement bien connu de Tristan et Iseut. Si l'on parle de l'intimité dans la littérature médiévale, il est en effet difficile d'omettre l'histoire de ce couple, d'autant plus pour un médiéviste qui ne dispose que de deux récits arthuriens en vieux tchèque et dont l'attention est sans doute attirée davantage par celui qui appartient à la matière tristanienne. On a vu que le conte Tandariáš a Floribella était une œuvre relativement courte, légère et enjouée, dont les protagonistes se créent des obstacles eux-mêmes, comme si le seul but était de divertir le lecteur curieux de voir comment les héros vont surmonter leurs difficultés. En revanche, le roman Tristram a Izalda semble au premier regard beaucoup plus grave.

Nous en connaissons deux versions manuscrites qui datent de 1449 et 1483. Chacune compte presque neuf mille vers. Du point de vue stylistique, ce texte présente des types de discours beaucoup plus variés, avec des modalités d'expression sensiblement plus riches. Le récit supporte sans aucun doute une lecture à plusieurs niveaux. Du point de vue de ses caractéristiques fondamentales, la narration ne s'éloigne pas des autres histoires tristaniennes médiévales - bien au contraire, il s'agit à proprement

33. Du point de vue anthropologique, voir M. IzARD, «Transgression, transversalité, errance», dans M. IzARD et P. Smith éd., La Fonction symbolique. Essais d'anthropologie, Paris, 1979, p. 289-306, ici p. 301 
parler d'une synthèse habile d'au moins trois versions allemandes: celle de Gottfried de Strasbourg, celle d'Heinrich de Freiberg et, en premier lieu, celle d'Eilhart d'Oberg de la fin du XII ${ }^{\mathrm{e}}$ siècle. En décidant ce qu'il doit incorporer ou non dans sa «traduction composée», l'anonyme tchèque met en valeur avec un intérêt évident quelques éléments qui concernent la présente étude des représentations de l'intime.

Il a profité entre autres du large champ symbolique du lit qui est, avant tout, un attribut de l'autorité royale. Ce motif était déjà présent dans des scènes bibliques où s'enracine une distinction sociale élémentaire entre le lit royal solide, tel qu'il apparaît dans l'Ancien Testament, et les simples couches portables que l'on pourrait simplement «prendre, [puis] se lever et marcher [avec]» dans les milieux pauvres du Nouveau Testament ${ }^{34}$. Si les moines trouvaient leurs modèles dans la pauvreté apostolique et ses modestes litières, les rois du Moyen Âge central, eux, se référaient à leurs prédécesseurs vétéro-testamentaires avec des pièces de mobilier coûteuses et richement décorées. On peut ainsi plus aisément comprendre, dans cette perspective, la remarque de Marie de France sur les décorations du lit «à l'uevre Salemun » dans le lai de Guigemar ${ }^{35}$. Bien entendu, c'est surtout le bateau qui attire ici l'attention du lecteur - mais cette constellation tripartite (le roi Salomon, le bateau, le lit-la tradition du pouvoir,le moyen de transport «physique», le moyen de transport « mental $\left.^{36} »\right)$ se trouve aussi bien dans le cycle Lancelot-Graal (et partiellement dans le roman Partonopeu de Blois). Chrétien de Troyes a lui aussi mis en scène des lits si beaux qu'ils seraient convenables «même pour un comte ou pour un roi» - notamment vers la fin du Conte du Graal où il a soumis Gauvain à l'épreuve du Lit de Merveille ${ }^{37}$. Du point de vue anthropologique, l'aventure du lit royal où il faut s'asseoir fermement et bien réussir à surmonter tous les obstacles, représente ici la puissance sexuelle (et c'est ainsi que le lit au milieu de la salle devient également un symbole familial, dynastique - et peut-être également juridique, en s'associant à Salomon). Concernant le statut social, le lit est une démonstration de la fortune, puisque c'est un objet très onéreux, dont la somptuosité n'est jamais omise dans les descriptions littéraires ${ }^{38}$.

34. Mt 9, 6; Mc 2,9; Jn 5, 8. Ainsi le lit devient aussi le symbole du corps ( $c f$. J.CHEVALIER et A.GHEerbrant, Dictionnaire des symboles, Paris, 1969, p. 465).

35. Lais de Marie de France,éd. L. HARF-LANCNER et K. WARNKE, Paris, 1990, p. 35-37, v. 170-203 (mais voir aussi G. D. WEST, «L'uevre Salemon», Modern Language Review, 49 v. 170-203 (mais voir
(1954), p. 176-182).

36. Mais pas seulement mental - rappelons les civières sur lesquelles les chevaliers blessés sont très souvent portés dans la littérature arthurienne.

37. Chrétien de Troyes, Le Conte du Graal, v. 7633-7635 (Romans, p. 1167), rappelé plus tard dans quelques autres récits, comme Galeran de Bretagne: Galeran de Bretagne, éd. J. DufOuRnet, Paris, 2009, p. 374, v. 5024.

38. E. FARAL, Recherches sur les sources latines des contes et romans courtois du Moyen Âge, Paris, 1913, p. 40-341; J. BumKE, Courtly Culture. Literature and Society in the High
Dans la perspective du «merveilleux », le lit permet, tout comme le bateau, des voyages nocturnes, en rêves, vers l'au-delà, mais aussi dans le monde temporel ici-bas par l'acte d'amour et de conception, par l'accouchement. Il est également associé à la maladie et à la mort ${ }^{39}$

La présence d'un péril incompréhensible, lié psychologiquement aussi bien aux rêves qu'aux actes sexuels, est fondée sur l'impossibilité de maîtriser sa performance sociale et même de se défendre dans son sommeil. Cela est exprimé par les risques liés à la pratique de l'hospitalité ${ }^{40}$ ou par des lances enflammées qui peuvent occasionnellement tomber sur les chevaliers dormants ${ }^{41}$. Ajoutons à cela les tensions autour de l'intime et de l'espace privé contesté, dont les deux premiers romans de Chrétien de Troyes contiennent beaucoup d'exemples ${ }^{42}$. On pourrait continuer avec la situation, beaucoup plus proche de la matière tristanienne, qu'offre la relation socialement influente de Lancelot et Guenièvre ou le conflit auquel est confronté Yvain, le chevalier au Lion, entre les intérêts de la famille et les activités du monde. C'est surtout là que se manifeste le clivage déjà mentionné: il est souhaitable que le souverain soit heureux en privé, car son intimité fertilement et profondément vécue assure le bien commun à tout le pays. Cependant, il lui faut trouver un juste équilibre. Si nous en venons aux literies monastiques, on peut bien citer Bernard de Clairvaux: le couvent, d'après lui, est aussi une sorte de lit où il est incontestablement bon ou même pieux de demeurer dans une union mystique avec le Christ. Mais il faut apprendre à en sortir, vers le monde extérieur, pour aider les autres ${ }^{43}$. Le moine cistercien combat pour ce même équilibre, comme par exemple Érec ou Yvain.

Middle Ages, Berkeley-Los Angeles-Oxford, 1991,p. 119-120.

39. M.-L.CHÊNERIE, Le Chevalier errant dans les romans arthuriens en vers des XII et XIII siècles, Genève, 1986, p. 85-86, p. 540-547 et 634-638; J.-C. PAYEN et H.Legros, «La femme et la nuit, ou recherche sur le thème de l'échange amoureux dans la littérature courtoise», dans Mélanges de langue et littérature françaises du Moyen Âge offerts à Pierre Jonin, Aix-en-Provence-Paris, 1979 (Sénéfiance, 7), p. 515-525

40. Voir Chrétien de Troyes, Érec et Énide, v. 3426-3457 (Romans, p. 169). Cela fait en effet trois nuits successives qu'Énide ne dort pas à cause de ses différentes craintes.

41. Chrétien de Troyes, Le Chevalier de la Charrette,v.503-519 (Romans, p. 515), dont l'interprétation christique a été proposée par J.RIBARD, Chrétiende Troyes, Le Chevalie de la Charrette. Essai d'une interprétation symbolique, Paris, 1972, p. 64-65; voir auss Sower and his Seed. Essays on Chrétien de Troyes, Lexington, 1983, p. 116-126.

42. Voir A.Мıсна, «Temps et conscience chez Chrétien de Troyes», dans ID., De la chanson de geste au roman. Études de littérature médiévale offertes par ses amis, élèves et collègues, Genève, 1976, p. 115-122.

43. Saint Bernard, Sermones super Cantica Canticorum, XLIV, 1-2 (Sancti Bernardi opera, éd. J. LeclercQ, H. Rochais et C. H. TAlBot, Rome, 1957, vol.2, p. 56-57). Le lit devient également une métaphore de l'union mystique: $c f$. É.GiLson, «La mystique de la grâce dans la Queste del Saint Graal», Romania, 51 (1925), p. 321-347. 
Revenons maintenant à l'intime dans le roman tchèque Tristram a Izalda. Bien sûr, cette histoire, comme les récits déjà mentionnés sur Tandariáš ou Lancelot, représente une collision provocante entre la sphère du mariage royal bénéfique et celle de l'amour merveilleux ${ }^{44}$, fondé explicitement, dans la légende tristanienne, sur la puissance extraordinaire du philtre. Ce qui s'oppose ici, c'est une intimité politiquement propice sous contrôle de la cour, et une intimité désordonnée, incontrôlable et difficilement compréhensible. Un peu comme un sanglier - qui plus est, destructeur lui aussi de l'ordre social. Ce motif a été justement repris par l'anonyme tchèque de la narration de Gottfried de Strasbourg ${ }^{45}$ :

Un sanglier effrayant et indomptable est sorti de la forêt. Il s'est dirigé vers la cour royale pour y provoquer un conflit. [...] Il est entré en courant là où se trouvait la chambre royale, dans laquelle le roi avec sa femme s'était retiré pour se coucher sur un lit. Il a défoncé la porte et fracassé en morceaux le lit où le roi même gisait. Il ne s'en est pas contenté : il a sali tout le lit avec sa salive et d'autres diverses saletés dégoûtantes.

Il s'agit ici d'un songe prophétique du sénéchal Marido. C'est une vive manifestation de ce que Tristram représente pour la cour et pour toute la société en entrant lui aussi, même si plus furtivement, dans la chambre royale. Cette destruction animale ne représente pas seulement la dévastation de l'union matrimoniale entre Izalda et le roi $\mathrm{Marek}^{46}$, mais aussi une contestation grave du pouvoir royal et, plus globalement, une déstabilisation de l'ordre social. C'est là que l'intimité du roi est étroitement liée à l'état du royaume - et Tristram ne possède évidemment pas les facultés salvatrices de Lancelot, pour lequel l'amour courtois pour la reine ne pose aucun obstacle à son service et à sa loyauté envers Arthur (y compris ses succès dans la tâche de rédemption du monde). En revanche, l'amour de Tristram et Izalda est proprement subversif. La destruction onirique du lit en est une représentation remarquable.

Bien que cela puisse être surprenant, on peut bien démontrer cette affirmation en évoquant une situation totalement inverse. En effet, si la présence du sanglier sale dans le lit du roi Marek présage la démolition du royaume, l'intrusion de Tristram dans l'intimité du couple royal n'est pas non plus anodine. Une fois gracié et glorifié, Tristram est autorisé à partager légalement la chambre du roi et de la reine, et participe ainsi publiquement

44. Sur les différents aspects de la notion du merveilleux, voir surtout J.LE GoFF, «Le merveilleux dans l'Occident médiéval», dans L'Imaginaire médiéval, Paris, 1985, p. 151187

45. Tristram a Izalda, éd. Z. TıснÁ, Prague, 1980, p. 44-45

46. J. M.FerRante, Tristan: A Comparative Study of Five Medieval Works, Ann Arbor, 1963, p. 103. à la pratique du pouvoir. Le roi Marek, après qu'il a cru que toutes les accusations d'adultère contre Tristram n'étaient que des calomnies, l'exprime clairement quand il ordonne ceci à Brangenena ${ }^{47}$ :

Prie le seigneur Tristram qu'il revienne, puisque toute la terre sera dans sa juridiction et soumise à lui. C'est à lui que tout le monde devra jurer obéissance. Je vais le prier, dans sa générosité, qu'il veuille me pardonner. Je ne veux plus reproduire les souffrances que je lui ai causées. Je vais installer son lit dans ma chambre. Ainsi, je le dédommagerai de son humiliation. De même, je veux bien qu'il demeure toujours avec la reine car j'ai bien vérifié qu'il n'a nullement touché à son honneur.

Bien sûr, le roi démontre ainsi que Tristram a regagné sa confiance et 1 peut donc lui ouvrir et rendre accessible son espace le plus privé, dans un geste de confiance suprême. De plus, il veut montrer nettement qu'il ne craint pas qu'une telle proximité avec la reine conduise à des abus. Il faut pourtant souligner qu'il veut également manifester ou même illustrer la part que prendra Tristram dans le gouvernement - de telle sorte qu'il l'invite à participer à l'intimité royale. Avec le pouvoir, il lui donne également accès à sa chambre ${ }^{48}$.

Mon cher cousin, veuille apporter ton lit dans ma chambre et rester là toujours. Ne quitte jamais la reine, en dépit de tout le monde qui te déteste ou qui ne supporte pas de te voir ici. Fais-le à leur déplaisir et sers toujours Sa Majesté

On a souvent interprété les implications de ce discours du roi de manière restrictive, comme si Tristram devenait seulement son chambellan d'honneur ${ }^{49}$. Sans vouloir contester l'importance de cette fonction à la cour, il me semble évident que Tristram gagne ici davantage en ce qui concerne le pouvoir et la participation au règne. La puissance symbolique du lit et de la chambre est ici beaucoup plus large ${ }^{50}$ :

Je vous ordonne à tous de ne pas négliger ce fait et d'obéir à Tristram comme à moi-même, d'accomplir toutes les fois sa volonté et de ne jamais rien lui opposer. Je suis désolé de l'avoir laissé tomber dans ma disgrâce. Tout cela s'est passé à cause d'une médisance et aujourd'hui je ne me le permettrai plus.

47. Tristram a Izalda, p. 55. On trouve un discours identique, provenant du texte de Béroul, dans la version d'Eilhart d'Oberg, le modèle principal du romancier tchèque.

48. Tristram a Izalda, p. 56

49. C. Marchello-Nizia éd., Tristan et Yseut : les premières versions européennes, Paris, 1995, p. 1619.

50. Tristram a Izalda, p. 55 
Il serait difficile d'exprimer plus clairement la relation forte et le rapport direct entre le règne et le lit comme attribut du règne. Cette exigence à propos de la transmission simultanée du pouvoir et de son symbole privilégié qu'est le lit est ensuite encore une fois répétée explicitement par Tristram ${ }^{51}$. Dans ce contexte, il ne me semble pas tout à fait extravagant de renvoyer de nouveau à la tradition vétérotestamentaire du lit de justice de Salomon ou bien aux récits féeriques des lits merveilleux ou périlleux. Partout, on peut apercevoir des indications de l'appartenance exclusive du lit au champ imaginaire des regalia secondaires. Pour le poète tchèque, de même que pour ses prédécesseurs allemands et français, le lit était un objet rare et précieux, très riche en fonctions et donc aussi en associations et significations, auxquelles nous ne pensons pas aujourd'hui. Cette perception, différente de la nôtre, se révèle dans les récits de fiction ${ }^{52}$.

L'intime comme thème historique n'est pas une invention à proprement parler postmoderne ${ }^{53}$. Les romanciers médiévaux ont eux aussi réfléchi délibérément sur l'espace privé comme tel. On a ici beaucoup parlé des chambres royales. Resterait à se demander comment les auteurs médiévaux eux-mêmes les percevaient. Est-ce qu'ils ont déjà noté un changement historique de la conception du privé? Suivons désormais la rencontre des rois Marek et Artuš, juste avant la scène nocturne fameuse où Tistram se blesse en mettant son pied sur une faux. La scène se déroule dans un passé fictif et très antérieur à l'époque de l'écriture du récit ${ }^{54}$ :

Je vous dirai assurément cela, qu'au temps jadis les rois se sont édifié et construit des maisons hautes et des palais très grands. Contrairement à aujourd'hui, ils ne s'intéressaient pas aux chambres, mais ils couchaient tous dans la salle. On peut donc observer que le roi Marc n'avait où se loger sinon au milieu de la grande salle du palais, avec tous ses invités. Eux s'y couchaient ainsi, parce qu'il n'y avait pas de chambre dans le palais.

Il paraît étonnant que l'on dise que les rois n'avaient pas de chambre, car c'est dans une chambre qu'on vient d'installer Tristram. Cependant, l'auteur de Tristram a Izalda, mais aussi Eilhart d'Oberg dont il a décidé de reprendre le constat, a compris que l'espace privé n'est qu'un privilège déterminé par le contexte historique. Cet espace privé était dédié à l'intime

51. Ibid., p. 56.

52. La richesse des fonctions et symboles associés au lit médiéval a été bien montrée lors de l'exposition récente à la Tour Jean sans Peur, dont on peut consulter le catalogue: D. AleXANDRe-Bidon, Au lit au Moyen Âge: catalogue de l'exposition, Paris, 2011.

53. G. Duby éd., Histoire de la vie privée, t. II, De l'Europe féodale à la Renaissance, Paris, 1985.

54. Tristram a Izalda, s. 71-72, voir le même constat dans la version d'Eilhart d'Oberg. mais également à la représentation de la fortune, de la famille, de la descendance et du pouvoir royal qui dispose de cet intime singulier. Les écrivains ont formulé la perception et l'exigence de l'intime comme une réalité qui ne cesse d'évoluer - de telle manière qu'ils ont eu besoin de la thématiser et de l'historiciser explicitement.

La littérature arthurienne médiévale dépeignait consciemment des conflits entre le privé et le public. Cela s'exprime à travers l'image littéraire du lit, où se matérialise le bonheur de l'intimité familiale, tout comme l'ennui causé par son absence. Le récit tchèque de Tristram met donc un accent fort sur l'aspect politique et social de l'espace privé, tandis que l'histoire de Tandariáš se concentre plutôt sur la description de l'état mental du chevalier, comprenant des images fréquentes de l'oubli de soi et de l'autodestruction amoureuse. Les deux récits prouvent qu'en Bohême, à la fin du XIv et au $\mathrm{XV}^{\mathrm{e}}$ siècle, il existait un public qui était déjà bien habitué des représentations raffinées, typiques de la littérature courtoise de l'Europe occidentale.

Martin šoRM - Centrum medievistických studií AV ČR (Centre d'études médiévales), Prague.

Représentations de l'intimité dans le roman arthurien tchèque

Les deux romans arthuriens tchèques de la fin du XIVesiècle (Tandariáš a Floribella et Tristram a Izalda) sont presentes comme des sources historiques sur la culture courtoise. Les auteurs médiévaux ont conçu les représentations littéraires de l'intime comme des moyens pour cultiver leur public et raffine leur sensibilité. Les scènes où le chevalier Tandariáš s'oublie lui-même et se blesse peuvent être comparées avec des motifs introduits par Chrétien de Troyes. L'intime se trouve aussi entre l'isolement dans l'espace privé et la performance sociale. Par exemple, le lit royal est présenté par l'auteur de Tristram a Izalda comme l'attribut du pouvoir. II témoigne de la manière dont Tristra in a zalda comme l'atribut du pouvoir '́émént concernant l'ense dont de la société.

culture courtoise - intimité - littérature arthurienne - lit - Tristan

Representations of Intimacy in the Czech Arthurian Romance

Two Czech Arthurian romances from the end of 14thcentury - Tandariás a Floribella and Tristram and Izalda - are presented as a historical source of courtly culture. Literary representations of intimacy were used by the authors to cultivate and refine the manners and thoughts of their aristocratic audience. For example, the scenes where the knight Tandariás forgets and wounds himself can be in this regard compared to some motifs introduced by Chrétien de Troyes. On the other hand, intimacy lies on the border between isolated privacy and social performance. For example the royal bed, used by the author of Tristram and Izalda as an attribute of power demonstrates how intimacy is considered of general interest to the whole society. Arthurian literature - bed - courtly culture - intimacy - Tristan 
